\title{
Sideband instability analysis based on a one-dimensional high-gain free electron laser model
}

\author{
Cheng-Ying Tsai, Juhao Wu, ${ }^{\dagger}$ Chuan Yang, ${ }^{\ddagger}$ Moohyun Yoon, ${ }^{\S}$ and Guanqun Zhou" \\ SLAC National Accelerator Laboratory, Menlo Park, California 94025, USA
}

(Received 8 August 2017; published 18 December 2017)

\begin{abstract}
When an untapered high-gain free electron laser (FEL) reaches saturation, the exponential growth ceases and the radiation power starts to oscillate about an equilibrium. The FEL radiation power or efficiency can be increased by undulator tapering. For a high-gain tapered FEL, although the power is enhanced after the first saturation, it is known that there is a so-called second saturation where the FEL power growth stops even with a tapered undulator system. The sideband instability is one of the primary reasons leading to this second saturation. In this paper, we provide a quantitative analysis on how the gradient of undulator tapering can mitigate the sideband growth. The study is carried out semianalytically and compared with one-dimensional numerical simulations. The physical parameters are taken from Linac Coherent Light Source-like electron bunch and undulator systems. The sideband field gain and the evolution of the radiation spectra for different gradients of undulator tapering are examined. It is found that a strong undulator tapering $(\sim 10 \%)$ provides effective suppression of the sideband instability in the postsaturation regime.
\end{abstract}

DOI: 10.1103/PhysRevAccelBeams.20.120702

\section{INTRODUCTION}

It is known that a free electron laser (FEL) is capable of generating coherent high-power radiation over a broad spectrum. Of particular interest is the atomic wavelength regime, e.g., from a fraction of a nanometer down to tenths of an angstrom, where the applications [1-3] typically require the radiation power in the terawatt (TW) range.

In a high-gain FEL, the main signal, which originates from the resonance condition, grows exponentially until saturation occurs. For the case of a constant or untapered undulator, the radiation power oscillates around an equilibrium in the saturation regime. In the x-ray FEL regime, the power efficiency (defined as the ratio of the FEL radiation power to the electron beam power) is about $10^{-3}$, indicating that the output peak power can be $\sim 50 \mathrm{GW}$ for an electron beam with peak current $\sim 5 \mathrm{kA}$ and energy $\sim 10 \mathrm{GeV}$ operating in the self-amplified spontaneous emission

\footnotetext{
*jcytsai@SLAC.Stanford.EDU

jhwu@SLAC.Stanford.EDU

Visiting Ph.D. student from National Synchrotron Radiation Laboratory, University of Sciences and Technology of China, No. 42 Hezuohua South Road, Hefei, Anhui 230029, China.

${ }^{\S}$ On sabbatical leave from Department of Physics, Pohang University of Science and Technology, Pohang 37673, Korea.

Visiting Ph.D. student from Institute of High Energy Physics, Chinese Academy of Sciences, and University of Chinese Academy of Sciences, 19B Yuquan Road, Shijingshan District, Beijing 100049, China.
}

Published by the American Physical Society under the terms of the Creative Commons Attribution 4.0 International license. Further distribution of this work must maintain attribution to the author(s) and the published article's title, journal citation, and DOI.
(SASE) mode in a 100-m-long untapered undulator. Though undulator tapering had been proposed since the 1980 s, recently there has been a renewed interest in tapering to achieve enhanced energy conversion efficiency, improved spectral purity, or for polarization control (see, for example, Refs. [4-9]). With undulator tapering, the efficiency can be improved and the power can be further increased in the postsaturation regime (however, at a lower rate compared with the exponential growth in the linear regime) but eventually will reach a so-called second saturation and the radiation then approaches another equilibrium. Although numerical simulations show that TW-level FEL output power can be possible when undulator tapering is optimized and the combined SASE and self-seeded scheme is employed (see, for example, Ref. [6]), in the postsaturation regime it is the sideband instability that still limits the growth of the main signal [4,10-13]. Enhancing the FEL peak power shall be envisioned once the sideband instability can be effectively suppressed.

The sideband instability in an FEL is caused by the interaction of the electromagnetic field with the electron synchrotron motion in the ponderomotive potential well. Such a potential well, formed by the undulator magnetic field and the main signal, will trap electrons and result in the oscillation with a synchrotron frequency (and its multiples) away from the resonance frequency (i.e., the frequency of the main signal). Once the interaction creates a positive feedback, the electron beam energy will transfer and contribute to the electromagnetic field with the synchrotron sideband frequency. The sideband signal will continue to grow and usually cause undesirable consequences. For example, it can degrade the spectral purity and may limit the level of the saturation power of FEL [6]. 
The early study of FEL sideband instability can be traced back to the 1980s $[4,10]$. The stability analyses are usually treated by single-particle or kinetic methods. Both approaches may assume small perturbations of electron synchrotron motion and radiation fields from the equilibria. While they may provide practical use to quickly estimate whether the sideband instability will be an issue, most of the previous work assumes constant-parameter undulators $[4,10,11]$, focuses on longer wavelength regime $[12,14,15]$, or addresses oscillator configurations [16,17]. Recently Lindberg [18] and Zhang et al. [19] have theoretically investigated the FEL sideband instability induced by initial beam modulations, in which their studies focus on a mechanism in the linear or exponential regime with a constant undulator parameter. Thereafter the beam-modulation induced sidebands have been intensively studied with numerical $[20,21]$ and experimental [22] studies. We remind that their studies assume the sideband signal lies within the typical FEL gain bandwidth. Therefore both the main and sideband signals are exponentially amplified in the linear regime.

In this paper our study will focus on the sideband instability in a single-pass high-gain tapered FEL in the post-saturation regime based on the single-particle description in a one-dimensional (1-D) model. The validity of 1-D analysis assumes that the transverse size of the electron beam is large compared to that of the radiation field, thus ignoring the effects of diffraction and the gradient of transverse electron beam density. The 1-D analysis captures the essence of sideband effects and thus can provide a quick estimate for sideband growth. Using the single-particle approach, we can obtain the corresponding dispersion equation, which accounts for sideband-related dynamical quantities. Then we derive the analytical expressions for two extreme cases: the gentle and strong undulator tapering. By quantifying the so-called sideband field gain, we find good agreement from theoretical predictions with results from our 1-D FEL simulations.

In the remainder of the paper, we first briefly introduce the 1-D FEL model in Sec. II A. In the derivation we follow the notations of Bonifacio et al. [23] and Isermann and Graham [24]. Our theoretical formulation begins at the first or initial saturation, where the information of the electron beam and radiation field shall be provided. The linear stability analysis is studied in Sec. II B based on the singleparticle description, assuming that the electron beam is deeply trapped in the FEL ponderomotive potential well. The perturbations on the electron phase space dynamics and field dynamics (amplitude and phase) are assumed small and included only up to first order to the unperturbed equilibria. The set of equations can then be formulated as a linear system and the stability is determined by the corresponding dispersion relation. In Sec. II C, we particularly focus on the effect of undulator tapering on the sideband growth and study both the gentle and strong undulator tapering and compare with the untapered case. Then in Sec. III we compare the semi-analytical calculations with full 1-D FEL numerical simulations based on similar parameters to those of the Linac Coherent Light Source (LCLS). The results confirm the effectiveness of strong undulator tapering on sideband suppression. Finally we summarize the results and discuss possible future work in Sec. IV.

\section{THEORETICAL FORMULATION}

The model we shall consider is based on the 1-D highgain FEL with a tapered planar undulator. Our primary focus will be in the post-saturation regime. The main signal, governed by the fundamental resonance condition, Eq. (1) below, is amplified through the high-gain FEL process, in which the electron beam energy will be transferred to the radiation field of the main signal along an undulator. In this equation, $\lambda_{u}$ is the undulator period, $\lambda_{R}$ is the radiation wavelength of the main signal, $\gamma_{R}(0)$ is the initial electron reference energy in units of its rest mass energy, $K_{0}$ is the (peak) untapered undulator parameter with $K_{0} \approx 0.934 B_{0}\left[\right.$ Tesla] $\lambda_{u}[\mathrm{~cm}]$ with $B_{0}$ being the peak undulator magnetic field

$$
\lambda_{R}=\frac{\lambda_{u}}{2 \gamma_{R}^{2}(0)}\left(1+\frac{K_{0}^{2}}{2}\right)
$$

Equation (1) can be obtained through the following two processes: the length contraction via Lorentz transformation of the undulator period from the laboratory frame to the electron rest frame and the Doppler effect converting the electron quiver motion in its rest frame back to the laboratory frame. In the presence of undulator tapering, the undulator parameter is in general a function of the undulator axis $z$, i.e., $K_{0} \rightarrow K(z)$. Here we only consider the $K$-tapering and assume $\lambda_{u}$ is constant. The maximized energy extraction roughly corresponds to a quadratic taper profile along the undulator axis, i.e., $\Delta K / K \propto z^{2}$ [4,5,7].

In the beam rest frame, the near-resonant electrons are trapped in the so-called ponderomotive potential well, in which these electrons execute a synchrotron motion around a synchronous particle. Such a ponderomotive potential well is formed by the undulator magnetic field and the radiation field. It is expected that the synchrotron motion will become appreciable once the FEL radiation field is saturated. The synchrotron motion in an FEL is similar to that in a storage ring, where the potential well created by a rf cavity causes the recirculating particles to execute a longitudinally periodic oscillation. In an FEL the synchrotron oscillation frequency is, however, usually larger than the transverse betatron oscillation, which results from the alternating-gradient quadrupole focusing (or defocusing) within undulator breaks. This is not the case in a storage ring. In what follows we neglect the betatron motion and 
our sideband study starts at the FEL first saturation, i.e., following the FEL exponential growth.

\section{A. Basic equations}

In the following we consider a general tapering profile of the undulator magnetic field,

$$
B(z)=B_{0} f_{B}(z),
$$

where $f_{B}(z)$ is the tapering profile of the undulator magnetic field. Equation (2) is equivalent to $K(z)=$ $K_{0} f_{B}(z)$. Then the 1-D FEL process can be formulated based on the following single-particle FEL Hamiltonian function averaged over the undulator period [23],

$$
\mathcal{H}(\theta, \eta ; \hat{z})=\frac{\left(\eta-\eta_{R}\right)^{2}}{2 f_{R}}-i \frac{f_{B}}{f_{R}}\left(\mathcal{E} e^{i \theta}-\mathcal{E}^{*} e^{-i \theta}\right),
$$

where $\theta=\left(k_{R}+k_{u}\right) z-\omega_{R} t$ is the electron phase with respect to the radiation, $\eta \equiv\left[\gamma-\gamma_{R}(0)\right] / \rho \gamma_{R}(0)$ is the normalized energy deviation with respect to the dimensionless FEL or Pierce parameter:

$$
\rho=\frac{1}{\gamma_{R}(0)}\left(\frac{\omega_{p b} K_{0} / \sqrt{2}}{4 c k_{u}}\right)^{2 / 3},
$$

with $\omega_{p b}=\sqrt{4 \pi e^{2} n_{0} / m_{0}}, e$ the charge unit, $n_{0}$ the volume density of the electron beam, $m_{0}$ is the electron rest mass. $\eta_{R}=\left[\gamma_{R}-\gamma_{R}(0)\right] / \rho \gamma_{R}(0)$. In the case of undulator tapering, the electron reference energy is modified accordingly through the resonance condition [see Eq. (1)] $\gamma_{R}(z)=$ $\gamma_{R}(0) f_{R}(z)$ where $f_{R}(z)=\sqrt{\frac{1+K^{2}(z) / 2}{1+K_{0}^{2} / 2}}$. Here $|\mathcal{E}|=|E| /$ $\sqrt{4 \pi n_{0} \rho \gamma_{R}(0) m_{0} c^{2}}$ is the normalized amplitude of the electric field $E$. Other relevant quantities are normalized as $\hat{z}=2 k_{u} \rho z, \quad \hat{s}=2 k_{u} \rho\left(z-\bar{\beta}_{z} c t\right) \quad$ with $k_{u}=2 \pi / \lambda_{u}$, $k_{R}=2 \pi / \lambda_{R}, \omega_{R}=c k_{R}$, and $\bar{\beta}_{z}=\bar{v}_{z} / c$. Moving to the ponderomotive frame, we define $\hat{u}=\hat{s} /\left(1-\bar{\beta}_{z}\right)$. The bars on $\beta_{z}$ and $v_{z}$ take the average over the electron's longitudinal figure- 8 motion.

From Hamilton's equations of motion, we have the electron phase equation

$$
\frac{d \theta}{d \hat{z}}=\frac{\partial \mathcal{H}}{\partial \eta}=\frac{\eta-\eta_{R}}{f_{R}}
$$

and the electron energy equation, relating the radiation field to

$$
\frac{d \eta}{d \hat{z}}=-\frac{\partial \mathcal{H}}{\partial \theta}=-\frac{f_{B}}{f_{R}}\left(\mathcal{E} e^{i \theta}+\mathcal{E}^{*} e^{-i \theta}\right) .
$$

Assuming $\Theta=\theta+\phi$, we have for the particle with reference energy $\gamma_{R}(\hat{z})$

$$
\frac{d \eta_{R}}{d \hat{z}}=-2 \frac{f_{B}}{f_{R}}\left|\mathcal{E}_{0}\right| \cos \Theta_{R},
$$

where $\mathcal{E}_{0}=\left|\mathcal{E}_{0}\right| e^{i \phi}$ with $\phi$ the phase of the radiation field and $\Theta_{R}=\theta_{R}+\phi$. In order for the electron to have a decrease of energy at resonance, we require $\Theta_{R} \in$ $[-\pi / 2, \pi / 2]$. For an untapered FEL, the reference phase is assumed to be fixed at $\Theta_{R}=-\pi / 2$.

For particles near the resonant electron, i.e., $\theta=\theta_{R}+\delta \theta$, combining Eq. (5) with Eq. (6) we get

$$
\frac{d^{2}}{d \hat{z}^{2}} \delta \theta+\Omega_{\mathrm{syn}, 0}^{2} \delta \theta=0
$$

where

$$
\Omega_{\mathrm{syn}, 0}^{2}=-2 \frac{f_{B}}{f_{R}^{2}}|\mathcal{E}| \sin \Theta_{R} .
$$

From Eq. (9), it is clear that those near-resonant electrons will execute an oscillation with the synchrotron frequency $\Omega_{\text {syn }, 0}$.

The energy transfer also involves the radiation field. For a number of individual electrons which follow from Eq. (3) and the Hamilton's equation, we have

$$
\frac{d \mathcal{E}}{d \hat{z}}=\left(\frac{\partial}{\partial \hat{z}}+\frac{\partial}{\partial \hat{u}}\right) \mathcal{E}=\frac{f_{B}}{f_{R}}\left\langle e^{-i \theta}\right\rangle,
$$

where the bracket $\langle\ldots\rangle \equiv N^{-1} \sum_{i=1}^{N}(\ldots)$ represents the ensemble average over a slice, with $N$ the number of electrons in the slice.

Equation (10) can also be obtained by Maxwell's equations by taking the slowly varying envelope approximation or paraxial approximation [4]. Inserting $\mathcal{E}=\left|\mathcal{E}_{0}\right| e^{i \phi}$ into Eq. (10) gives two separate equations for the amplitude and phase of the radiation field. To the zeroth order we have

$$
\frac{d}{d \hat{z}}\left|\mathcal{E}_{0}\right|=\left(\frac{\partial}{\partial \hat{z}}+\frac{\partial}{\partial \hat{u}}\right)\left|\mathcal{E}_{0}\right|=\frac{f_{B}}{f_{R}}\langle\cos \Theta\rangle
$$

and

$$
\left|\mathcal{E}_{0}\right| \frac{\partial \phi}{\partial \hat{z}}=-\frac{f_{B}}{f_{R}}\langle\sin \Theta\rangle .
$$

In the case of untapered FEL, the field amplitude after saturation is considered to be constant and the radiation phase $\phi$ is linear in $z$. This observation is consistent with that discussed in Ref. [25]. In what follows, the main signal will be determined by the conservation of energy. From Eqs. (7) and (11), we have $d\left|\mathcal{E}_{0}\right| / d \hat{z} \approx \frac{-1}{2\left|\mathcal{E}_{0}\right|} d \eta_{R} / d \hat{z}$ assuming $\langle\cos \Theta\rangle \approx \cos \Theta_{R}$. Then the field amplitude can be expressed as

$$
\left|\mathcal{E}_{0}\right|=\sqrt{\left|\mathcal{E}_{0}^{(0)}\right|^{2}+\frac{\left(1-f_{R}(\hat{z})\right)}{\rho}},
$$


where use of $\eta_{R}(\hat{z})=\left(f_{R}(\hat{z})-1\right) / \rho$ has been made and $\left|\mathcal{E}_{0}^{(0)}\right|$ is the normalized field amplitude at the starting location of undulator tapering (denoting $\hat{z}=\hat{z}_{b}$ ) and considered as a given parameter.

Note that $\Theta_{R}$ is not necessarily a constant over $\hat{z}$. In our analysis a taper profile function $f_{B}(\hat{z})$ is assumed to be given. Then $f_{R}(\hat{z}), f_{R}^{\prime}(\hat{z})$, and $\eta_{R}^{\prime}=f_{R}^{\prime} / \rho$ can be obtained. From Eq. (7) $\Theta_{R}$ is determined. For untapered case, $\Theta_{R}$ is a constant, $-\pi / 2$.

\section{B. Linear stability analysis}

In the previous subsection we have obtained particle equations [Eqs. (5) and (6)] and field equations [Eqs. (10)(12)]. Now we can study the stability of the 1-D FEL system. Since we are interested in the sideband instability after the first saturation, we will Taylor expand the dynamical quantities around their saturation equilibria and study how the perturbation is affected by the dynamical system. Let us assume

$$
\begin{aligned}
\mathcal{E} & =\left(\left|\mathcal{E}_{0}\right|+\delta \mathcal{E}^{\prime}+i \delta \mathcal{E}^{\prime \prime}\right) e^{i \phi} \\
\eta & =\eta_{R}+\delta \eta \\
\theta & =\theta_{R}+\delta \theta,
\end{aligned}
$$

where $\theta_{R}=\Theta_{R}-\phi$. The quantities denoted with $\delta$ are considered to be small and sideband-related quantities. Once these small perturbed quantities are comparable to their unperturbed values, the linear or first-order analysis no longer becomes valid and higher-order effects will come into play. In this paper, however, we content ourselves with the first order analysis because the sideband instability growth at the onset is our primary interest.

Next, because we are linearizing the dynamical system, we assume these perturbations do not interact with each other and behave as $\left(\delta \theta, \delta \eta, \delta \mathcal{E}^{\prime}, \delta \mathcal{E}^{\prime \prime}\right) \propto \operatorname{Re}\left(e^{i k \hat{z}-i \kappa \hat{u}}\right)$, where $\kappa$ is assumed to be real and $k$ can be in general complex. The real part of $k$ represents the propagation constant, while the imaginary part indicates the growth (or damping) of the associated quantities. Inserting Eq. (14) into Eqs. (5), (6) and (10) and taking the ensemble average to first order $\left\langle e^{-i \delta \theta}\right\rangle=1-i\langle\delta \theta\rangle$ will result in

$$
\begin{gathered}
\frac{d}{d \hat{z}} f\langle\delta \theta\rangle-\frac{1}{f_{R}}\langle\delta \eta\rangle=0, \\
\frac{d}{d \hat{z}}\langle\delta \eta\rangle-2 \frac{f_{B}}{f_{R}}\left[\left|\mathcal{E}_{0}\right| \sin \Theta_{R}\langle\delta \theta\rangle-\cos \Theta_{R}\left(\delta \mathcal{E}^{\prime}\right)\right. \\
\left.+\sin \Theta_{R}\left(\delta \mathcal{E}^{\prime \prime}\right)\right]=0, \\
\left(\frac{\partial}{\partial \hat{z}}+\frac{\partial}{\partial \hat{u}}\right)\left(\delta \mathcal{E}^{\prime}\right)+\frac{f_{B}}{f_{R}\left|\mathcal{E}_{0}\right|} \sin \Theta_{R}\left(\delta \mathcal{E}^{\prime \prime}\right) \\
+\frac{f_{B}}{f_{R}} \sin \Theta_{R}\langle\delta \theta\rangle=0,
\end{gathered}
$$

$$
\begin{gathered}
\left(\frac{\partial}{\partial \hat{z}}+\frac{\partial}{\partial \hat{u}}\right)\left(\delta \mathcal{E}^{\prime \prime}\right)-\frac{f_{B}}{f_{R}\left|\mathcal{E}_{0}\right|} \sin \Theta_{R}\left(\delta \mathcal{E}^{\prime}\right) \\
+\frac{f_{B}}{f_{R}} \cos \Theta_{R}\langle\delta \theta\rangle=0 .
\end{gathered}
$$

In obtaining Eqs. (17) and (18) we have employed Eqs. (11) and (12). The above four linearized equations can be put in an algebraic form after decomposing into the frequency domain

$$
\begin{aligned}
& \left(\begin{array}{cccc}
i k & -f_{R}^{-1} & 0 & 0 \\
f_{R} \Omega_{\mathrm{syn}, 0}^{2} & i k & 2 \frac{f_{B}}{f_{R}} \cos \Theta_{R} & \frac{f_{R}}{\left|\mathcal{E}_{0}\right|} \Omega_{\mathrm{syn}, 0}^{2} \\
-\frac{f_{R}}{2\left|\mathcal{E}_{0}\right|} \Omega_{\mathrm{syn}, 0}^{2} & 0 & i(k-\kappa) & -\frac{f_{R}}{2\left|\mathcal{E}_{0}\right|^{2}} \Omega_{\mathrm{syn}, 0}^{2} \\
\frac{f_{B}}{f_{R}} \cos \Theta_{R} & 0 & \frac{f_{R}}{2\left|\mathcal{E}_{0}\right|^{2}} \Omega_{\mathrm{syn}, 0}^{2} & i(k-\kappa)
\end{array}\right) \\
& \times\left[\begin{array}{l}
\langle\delta \theta\rangle \\
\langle\delta \eta\rangle \\
\delta \mathcal{E}^{\prime} \\
\delta \mathcal{E}^{\prime \prime}
\end{array}\right]=\mathbf{0} .
\end{aligned}
$$

Now the stability of such a linear system is determined by the properties of the matrix. Existence of nontrivial solutions require that the corresponding determinant of the matrix vanishes. Then we have the following dispersion relation

$$
\begin{gathered}
\left(k^{2}-\Omega_{\mathrm{syn}, 0}^{2}\right)\left[(k-\kappa)^{2}-\frac{f_{R}^{2}}{4\left|\mathcal{E}_{0}\right|^{4}} \Omega_{\mathrm{syn}, 0}^{4}\right] \\
-\frac{f_{B}^{2}}{f_{R}^{2}\left|\mathcal{E}_{0}\right|^{2}} \Omega_{\mathrm{syn}, 0}^{2}=0 .
\end{gathered}
$$

This dispersion relation has been derived in the existing literature, e.g., Ref. [10-12,24]. In this equation we will solve for $k$ provided $\kappa$ is given. From Eq. (20) it is obvious that $k(-\kappa)=-k(\kappa)$. It is worth pointing out that in our analysis the spectrum of $k(\kappa)$ is symmetric in $\kappa$, which means that the growth rates of both lower and upper sidebands are equal. However in the numerical FEL simulations the output radiation spectrum after saturation usually consists of more lower sidebands than upper sidebands because the electron beam is slightly detuned and the favored electron beam is usually above resonance (see, for example, Ref. [26] for further discussion). More specifically, whether the lower or upper sideband dominates (or both) depends on the detailed electron phase space distribution in action space [26]. In the following we restrict ourselves to the discussion of the dominant sideband, which is usually, but not necessarily, the lower one. As a matter of fact, in our studies we find that the predicted results from Eq. (20) correspond well to the dominant sideband. Note that in Eq. (20) the relevant quantities are 
basically $z$-dependent in the presence of undulator tapering. Note also that in our theory the dynamical equations [i.e. Eqs. (15)-(18)] are linearized, which means that in our discussion the sideband growth will only behave exponentially and no longer saturate. The linear or exponential growth of the sideband signal(s) is of our primary concern and the study of the sideband saturation is beyond the scope of the analysis.

While there exist some approximate analytical solutions $[10,24]$, in the most general case the dispersion equation needs to be solved numerically. We will have a brief discussion in the next subsection on the approximate analytical solutions and in Sec. III compare the semianalytical and numerical calculations of the dispersion relation with the full 1-D FEL numerical simulation.

\section{Semianalytical results}

For Eq. (20), the behavior of the imaginary part of $k$, $\operatorname{Im} k<0$, features the growth of the sideband signal, where the maximum growth rate occurs at $\kappa \approx \Omega_{\mathrm{syn}, 0}(\hat{z})$. The general characteristics of the dispersion curve is shown in Fig. 1. There exists a cutoff threshold for $\kappa$, above which the oscillating electron beam will not interact with the corresponding spectral components and the corresponding signals do not exist.

Now let us try to find an analytical expression for the maximum growth rate, i.e. we will look for $k=$ $\Omega_{\mathrm{syn}, 0}+\delta k$, where $\delta k$ is in general a complex quantity. Inserting it into Eq. (20) and letting $d^{2} \delta k / d \kappa^{2}=0$, we can obtain an approximate expression for the instantaneous maximum growth rate $[10,24]$

$$
\max |\operatorname{Im} k| \approx \frac{\sqrt{3}}{2}\left[\frac{f_{B}^{2}(\hat{z}) \Omega_{\mathrm{syn}, 0}(\hat{z})}{2 f_{R}^{2}(\hat{z})\left|\mathcal{E}_{0}(\hat{z})\right|^{2}}\right]^{1 / 3} .
$$

Here the $\hat{z}$-dependence is explicitly attached for clarity. This expression may still be less practical because, to evaluate Eq. (21), many pieces of $\hat{z}$-dependent information

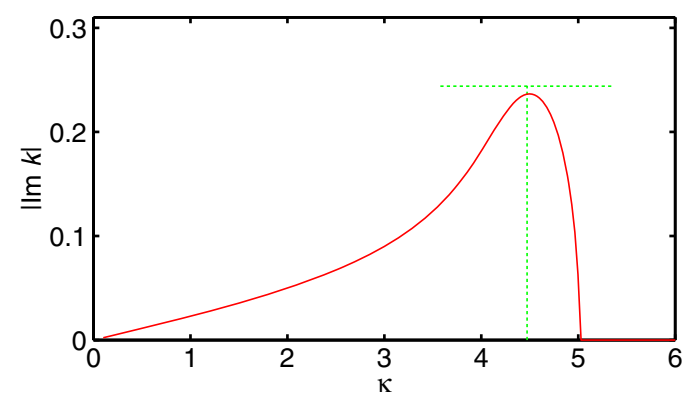

FIG. 1. The growth rate $|\operatorname{Im} k|$ as a function of $\kappa$. The dispersion curve is obtained by solving Eq. (20) with $\Omega_{\text {syn }, 0} \approx 4.4,\left|\mathcal{E}_{0}\right| \approx 10$, and $f_{B}=f_{R}=1$. The green dashed lines indicate the analytical approximate solutions of the maximum growth rate $\max |\operatorname{Im} k|$ [Eq. (21)] at $\kappa \approx \Omega_{\text {syn }, 0}$. should be given in advance. Let us further simplify Eq. (21) and express the maximum sideband growth rate in terms of quantities at $\hat{z}=\hat{z}_{b}$, where $\hat{z}_{b}$ is the starting location of the undulator tapering. We will separate the following discussion into two cases: the gentle and strong undulator tapering. Let us define the taper ratio $\Delta$ such that $f_{B}(\hat{z})=1-\Delta(\hat{z})$. For gentle tapering the taper ratio $\Delta \ll$ $\rho\left|\mathcal{E}_{0}^{(0)}\right|^{2}<1$ is assumed. The existing LCLS undulator tapering with $\Delta=0.8 \%$ is an example of this case (where $\rho \approx 1.57 \times 10^{-3}$ and $\left|\mathcal{E}_{0}^{(0)}\right| \approx 2.52$ ). For $\Delta \ll 1$, we have $f_{B}^{2} \approx 1-2 \Delta, f_{B} / f_{R} \sim 1-\Delta, \Omega_{\mathrm{syn}, 0} \approx \Omega_{\mathrm{syn}, 0}^{(0)}\left(1+\Delta / 4 \rho\left|\mathcal{E}_{0}^{(0)}\right|^{2}\right)$ with $\Omega_{\text {syn, } 0}^{(0)}$ the synchrotron frequency at $\hat{z}=\hat{z}_{b}$ for the untapered case, and $\left|\mathcal{E}_{0}\right| \approx\left|\mathcal{E}_{0}^{(0)}\right|\left(1+\Delta / 2 \rho\left|\mathcal{E}_{0}^{(0)}\right|^{2}\right)$. Then Eq. (21) can be further simplified to be

$$
\begin{gathered}
\max |\operatorname{Im} k|_{\text {gentle taper }} \approx \max |\operatorname{Im} k|_{\text {un-tapered }} \\
\times\left(1-\frac{1}{2} \xi+\frac{5}{8} \xi^{2}-\frac{15}{16} \xi^{3}+\cdots\right)
\end{gathered}
$$

where $\xi=r\left[K_{0}^{2} /\left(4+2 K_{0}^{2}\right)\right], \quad r=\Delta / \rho\left|\mathcal{E}_{0}^{(0)}\right|^{2}, \quad$ and the instantaneous sideband growth rate for the untapered case is $\max |\operatorname{Im} k|_{\text {un-tapered }} \approx \frac{\sqrt{3}}{2}\left(\Omega_{\text {syn, } 0}^{(0)} / 2\left|\mathcal{E}_{0}^{(0)}\right|^{2}\right)^{1 / 3}$.

From Eq. (22) it is interesting to see that the undulator tapering to some extent can reduce the sideband growth. Note that Eq. (22) is only valid for small ratios of undulator tapering (or $r \ll 1$ ). For strong undulator tapering the taper ratio is assumed to be $\rho\left|\mathcal{E}_{0}^{(0)}\right|^{2} \ll \Delta<1$. For example, $\Delta=15 \%$ with the aforementioned $\rho$ and $\left|\mathcal{E}_{0}^{(0)}\right|$. From Eq. (13) the main signal can now be approximated to be $\left|\mathcal{E}_{0}\right| \approx \sqrt{\Delta / \rho}\left(1+\rho\left|\mathcal{E}_{0}^{(0)}\right|^{2} / 2 \Delta\right)$, and the synchrotron frequency now becomes approximately $\Omega_{\mathrm{syn}, 0} \approx \Xi(1-\Delta / 2)$ where $\Xi^{2}=-2 \sqrt{\Delta / \rho} \sin \Theta_{R}$. Inserting into Eq. (21) results in

$$
\max |\operatorname{Im} k|_{\text {strong taper }} \approx \frac{\sqrt{3}}{2}\left(\frac{1}{2} \Xi \frac{\rho}{\Delta}\right)^{\frac{1}{3}}\left[1-\frac{5}{6}\left(\Delta-\rho\left|\mathcal{E}_{0}^{(0)}\right|^{2}\right)\right] .
$$

From Eq. (23) one can see that, while stronger undulator tapering can help reduce the sideband growth, it also effects the accompanying (growing) main signal and will make the reduction become ineffective. Figure 2 shows the $z$-dependence of the maximum sideband growth rate for two different taper ratios (or saturation main signals): $0.1 \%$ undulator tapering $(\Delta=0.001$ or $r=0.1)$ and $20 \%$ undulator tapering $(\Delta=0.2$ or $r=20)$. One can see that the larger the ratio of undulator tapering (or the stronger the main signal), the more reduced the instantaneous sideband growth rate will be. The growth rate for strong undulator 


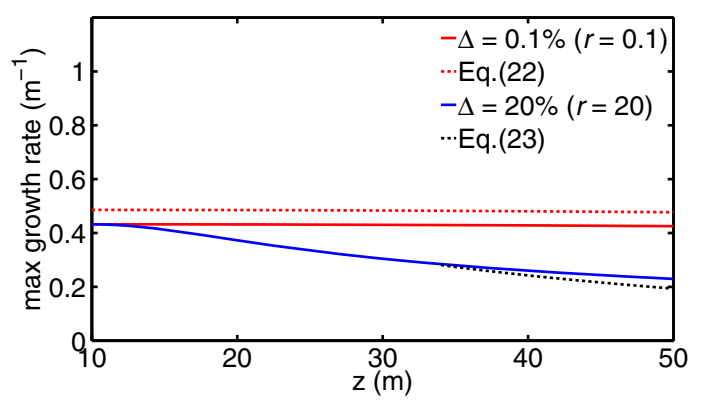

FIG. 2. Sideband growth rate as a function of $z$ for two cases: the gentle and strong undulator tapering. The red solid line refers to the gentle tapering and is obtained by numerically solving Eq. (20) and taking the maximum value from the dispersion curve at each $z$ location; the red dotted line is calculated from Eq. (22) for $\Delta=0.1 \%$. The blue solid and black dotted lines refer to the strong tapering, in which the former is obtained from Eq. (20) and the latter from Eq. (23). In the black dotted line, $\Xi \approx 4.7$ at $z \approx 50 \mathrm{~m}$. Here $\rho \approx 1.57 \times 10^{-3}$ and $\left|\mathcal{E}_{0}^{(0)}\right| \approx 2.52$.

tapering will however gradually approach that predicted by Eq. (23).

Before ending this section we note that the theoretical analysis is based on the single-particle description [10,24], i.e. the electrons executing synchrotron oscillations are assumed deeply trapped around the bottom of the potential well. Such a treatment has excluded possible asymmetric sidebands from the radiation spectrum. It turns out that the theoretical prediction in fact corresponds to the dominant sideband in the next section. We also comment that although the further simplified expressions, Eqs. (22) and (23) [compared with Eq. (21)], may restrict the range of validity, they should lead to clearer, approximate predictions and better insight into the FEL sideband growth.

\section{COMPARISON WITH 1-D FEL NUMERICAL SIMULATIONS}

In Sec. II we have derived the dispersion relation for the FEL sideband instability growth. Now we will compare the semianalytical and numerical solutions of Eq. (20) with the full 1-D FEL simulation results. The 1-D FEL simulation numerically solves Eqs. (5), (6), and (10) in a selfconsistent manner. In this code the shot noise is generated based on Penman-McNeil algorithm [27] and Fawley beamlet scheme [28] and span to $2 \pi$ for initial particle phases of a beamlet. Each beamlet consists of 4 macroparticles. Within a beamlet the particle energies are the same. Among beamlets (which form a bunch slice), the particle energies are sampled based on Gaussian distributed pseudo-random number. The simulation evolves each bunch slice (from tail to head) through the undulator sections. Numerical implementation of the radiation advection between electron beam slices is made by applying the appropriate discrete slippage. The interested reader is referred to Ref. [29] for introduction of the numerical
TABLE I. Numerical parameters for the beam, undulator and radiation fields for the hard $\mathrm{X}$-ray FELs.

\begin{tabular}{lcc}
\hline \hline Name & Value & Unit \\
\hline Electron beam energy & 10.064 & $\mathrm{GeV}$ \\
RMS relative energy spread & $10^{-4}$ & \\
Peak current & 4 & $\mathrm{kA}$ \\
Normalized emittances $(x, y)$ & $0.3,0.3$ & $\mu \mathrm{m}-\mathrm{rad}$ \\
Average beta function $(x, y)$ & 5,5 & $\mathrm{~m}$ \\
Undulator parameter $K_{0}$ (peak) & 3.5 & \\
Undulator period & 3 & $\mathrm{~cm}$ \\
Input seed power & 1 & $\mathrm{MW}$ \\
Resonance wavelength & $2.755 / 4.5$ & $\AA / \mathrm{keV}$ \\
First saturation power & $\sim 80$ & $\mathrm{GW}$ \\
First saturation length & $\sim 13$ & $\mathrm{~m}$ \\
\hline \hline
\end{tabular}

algorithms. Table I summarizes the relevant beam, undulator, and FEL parameters for a hard x-ray seeded FEL configuration. The physical parameters are taken from an LCLS-like electron bunch and undulator system. The key numerical parameters include the mesh numbers for undulator $z$-axis and bunch internal $s$-axis. The grid size along $z$, $\Delta z$, is chosen that it should be smaller than the 1-D FEL gain length $L_{g}^{1 D} \approx \lambda_{u} / 4 \pi \rho$ and must be larger than the undulator period $\lambda_{u}$ (usually a multiple of $\lambda_{u}$ 's). The grid size along $s$-axis, $\Delta s$, is chosen so that it should both resolve at least one electron bunch slice in an integration step $\Delta z$ and satisfy the fundamental resonance condition. In our 1-D FEL simulation we choose $\Delta z \approx 0.15 \mathrm{~m}$, for the $\approx 50 \mathrm{~m}$ long undulator, $\Delta s \approx 10 \lambda_{u}$ with the whole bunch duration $7.2 \mu \mathrm{m}$, and 1024 macroparticles for each bunch slice.

To compare the theoretical prediction presented in Sec. II with the 1-D FEL simulation, we first need to analyze the FEL output spectra. In FEL simulations, the representative macroparticles of the electron beam will rotate in the longitudinal phase space $(\theta, \eta)$ [see also Eqs. (5) and (6)]. Such a rotation, i.e., synchrotron motion, will feature a finite energy spread, which will result in spread of the sideband spectrum [13]. For this we take the following steps to more reasonably account for the spectral width due to finite energy spread. First we define the trapping parameter as

$$
\lambda \equiv \frac{1}{2}\left(1-\frac{f_{R} \mathcal{H}}{2 f_{B}|\mathcal{E}|}\right),
$$

where $\mathcal{H}$ is introduced in Eq. (3).

From Eq. (3) it can be found that the trapping parameter $\lambda$ varies from 0 to 1 , corresponding to the electron located in the very bottom or at the stable boundary (i.e., separatrix) of the ponderomotive potential well, respectively. For simplicity, we assume the electrons in the beam are uniformly distributed in the longitudinal phase space bucket. 
The effective energy spread can be estimated to be $\mathcal{H}^{*}=$ $\left(\sigma_{\eta}-\eta_{R}\right)^{2} / 2 f_{B}$, where $\sigma_{\eta}$ is the relative energy spread normalized to $\rho$. This $\mathcal{H}^{*}$ corresponds to an effective trapping parameter $\lambda^{*}$ by Eq. (24). Then the synchrotron frequency corresponding to $\mathcal{H}^{*}$ will be [13]

$$
\Omega_{\mathrm{syn}}(\hat{z})=\frac{\pi \Omega_{\mathrm{syn}, 0}(\hat{z})}{2 E_{1}\left(\lambda^{*}\right)},
$$

where $\Omega_{\mathrm{syn}, 0}(\hat{z})$ is the synchrotron frequency obtained in Eq. (9), and $E_{1}$ is the complete elliptic integral of the first kind, defined as $E_{1}(\lambda)=\int_{0}^{\pi / 2} d \vartheta / \sqrt{1-\lambda \sin ^{2} \vartheta}$. At the bottom of the well, where $\lambda=0$ and $E_{1}(0)=\pi / 2$, we have $\Omega_{\text {syn }}(\hat{z})=\Omega_{\text {syn }, 0}(\hat{z})$. The synchrotron frequency gets smaller when a particle deviates farther from the synchronous one.

To quantify the sideband signal or sideband field around the sideband frequency, we evaluate

$$
\mathcal{E}_{s}^{ \pm}(\hat{z}) \approx \frac{1}{c \delta \Omega} \int_{\omega_{s}^{ \pm}}^{\omega_{s}^{ \pm} \mp c \delta \Omega}|\tilde{\mathcal{E}}(\omega)| d \omega,
$$

where the upper and lower signs correspond to the upper and lower sideband signal, respectively. $\delta \Omega=\Omega_{\mathrm{syn}, 0}(\hat{z})-$ $\Omega_{\text {syn }}(\hat{z})$ is the sideband width, $\omega_{s}^{ \pm}=\omega_{0} \pm \omega_{s}$, and $\omega_{s} \approx$ $2 \gamma_{R}^{2}(\hat{z}) c \Omega_{\mathrm{syn}, 0}(\hat{z})$ is the shift of the central sideband frequency from the main signal $\omega_{0}$.

Then we define the sideband field gain as

$$
G(\hat{z}) \equiv\left|\frac{\mathcal{E}_{s}(\hat{z})}{\mathcal{E}_{s}\left(\hat{z}_{b}\right)}\right|=e^{\Lambda(\hat{z})},
$$

where

$$
\Lambda(\hat{z})=\int_{\hat{z}_{b}}^{\hat{z}} \max |\operatorname{Im} k(\zeta)| d \zeta
$$

is the sideband field growth. For simplicity, the superscript \pm is neglected here. Equations (27) and (28) will be applied to both lower and upper sidebands.

In the remaining part of this section we will demonstrate an example based on LCLS-like parameters (see Table I) and investigate the impact of different levels of undulator tapering on the sideband instability. Then we will compare the theoretical predictions based on the dispersion equation, Eq. (20), with the results from 1-D FEL numerical simulations. Figure 3 shows the evolution of the FEL peak power as a function of $z$. In this work, we are mostly interested in the third (last) section of LCLS undulator system, which consists of 16 undulator segments (the 17th to $32 \mathrm{nd}$ undulators) with the total length about $52 \mathrm{~m}$. Each undulator section consists of 110 undulator periods with $\lambda_{u}=3 \mathrm{~cm}$. Within consecutive undulator sections the undulator breaks are not accounted for in our simulation.

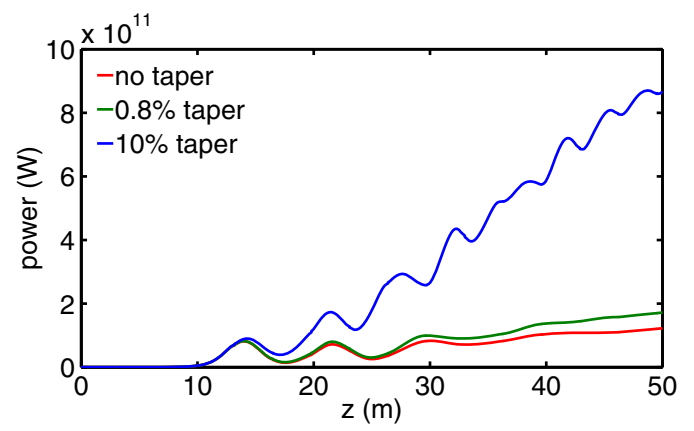

FIG. 3. FEL peak power as a function of $z$ for untapered (red), $0.8 \%$-tapered (green), and 10\%-tapered (blue) cases.

A step-wise undulator tapering with pure quadratic profile is assumed and begins at $z \approx 10 \mathrm{~m}$, i.e., from 20th undulator. Shown in Fig. 3 the initial saturation occurs at $z \approx 13 \mathrm{~m}$ for all three different cases: no taper $(\Delta=0)$, gentle taper $(\Delta=0.8 \%)$, and strong taper $(\Delta=10 \%)$. In the same figure we see that for $0.8 \%$ undulator tapering the final power increases about $40 \%$ compared to the untapered case. For an assumed 10\% undulator tapering (although LCLS does not have this option), the final power grows about seven times that of the untapered case. Here we do not intend to optimize the output power; therefore the starting location of the undulator tapering and the taper coefficients (with linear, quadratic or even with fractional order) are not optimized. Instead, we put the emphasis on sideband dynamics, particularly the spectral evolution in our analysis and in the subsequent simulations. The dip structure shown in Fig. 3 can be partially attributed by the unoptimized taper, i.e. the power saturates around $13 \mathrm{~m}$ but still oscillates until about $30 \mathrm{~m}$ and then starts to increase. This would suggest that the taper starting location is still a bit late, and it would be beneficial to begin the tapering even earlier to obtain higher peak power. Another reason for such a dip structure is that we use the ideal electron beam. Each slice of the beam will respond in a relatively coherent way, compared with the real beam, and will therefore evolve with more evident synchrotron motions in the longitudinal phase space. Such a synchrotron motion will lead to energy transfer between the radiation and the electron beam. These two reasons will lead to different outcomes that are usually reported in the literature (see, for example, Ref. [30]). Our theoretical analysis assumes the beginning of undulator tapering at $z \approx 10 \mathrm{~m}$. Note that the theory does not distinguish the beginning of undulator tapering from the saturation point. The 1-D gain length is about $1.52 \mathrm{~m}$ in the present example. While there is not a clear identification of saturation point, it is empirically suggested that the undulator tapering can begin at about two FEL gain lengths prior to the saturation [31].

Now let us look at the effect of different undulator taperings on the sideband instability gain. Figure $4(\mathrm{a}-\mathrm{c})$ corresponds to three different situations with no taper, $0.8 \%$, and $10 \%$ undulator tapering, respectively. From the 

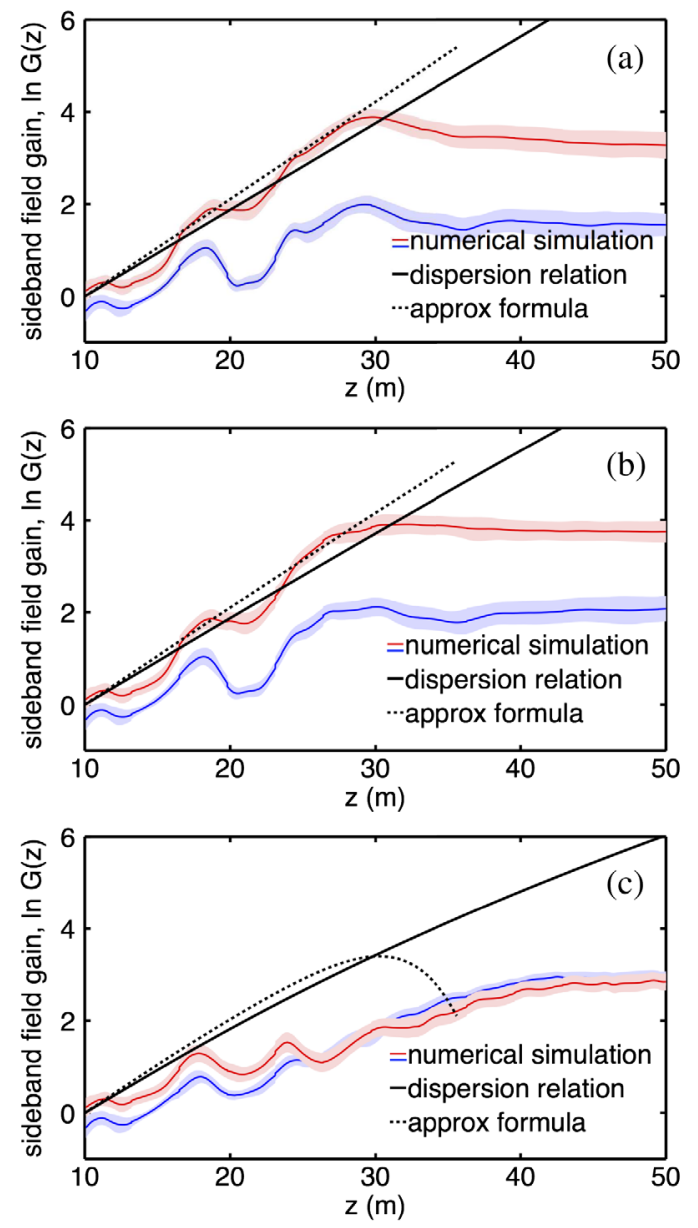

FIG. 4. Sideband field gain $\ln G(z)$ as a function of $z$ for (a) untapered, (b) 0.8\%-tapered, and (c) 10\%-tapered cases. The numerical simulations are averaged results from 50 independent runs. The solid lines are obtained from solving Eq. (20) and the dashed lines are evaluated by Eqs. (22) and (28). The shaded error bars, denoted as statistical fluctuations $( \pm 1 \sigma)$ from each independent 1-D FEL numerical simulations, are marked with red and blue and correspond to the lower and upper sideband signals, respectively.

figure we see that both the theoretical predictions and the 1-D FEL simulation results match reasonably well. In particular, we find that as the taper ratio increases, the sideband field gains become reduced, reflected in both reduction of the slopes of the black solid and dotted lines (theory) and those of red and blue curves (numerical simulations). Comparing simulation results in Fig. 4(a) and Fig. 4(b) with Fig. 4(c), we have an interesting observation that the lower sideband dominates in the untapered and gentle-tapered case while the lower and upper sideband fields become comparable in the strong undulator tapering. As mentioned in the previous section, the single-particle treatment, which assumes the electrons are deeply trapped around the bottom of the FEL ponderomotive potential well, will only give the symmetric spectrum for sideband growth. Thus the theoretical prediction can not distinguish whether the lower or upper sideband will dominate. The otherwise dominant sideband has always smaller sideband field growth and will be of less concern. Such an asymmetric sideband spectrum, either lower or upper sideband will dominate, can indeed depend on the detailed electron distribution in the phase space [13]. Furthermore, in a short-wavelength FEL an electron beam, composed of different longitudinal slices, may execute slightly different synchrotron motions, and will then respond to different portions of the sideband spectrum. More specifically, it can be possible that only partial, not the entire range, of the upper or lower sideband spectrum will dominate against the other. The present theoretical analysis simply assumes that electrons of all slices respond identically. This is one reason that the theoretical prediction overestimates the numerical simulation results. While our focus is the impact of undulator taper gradient on the sideband growth, we refer the interested reader to Refs. $[19,26]$ for the particular issue of asymmetric sideband growth. Another observation is the sideband saturation, where the sideband growth slows down or even ceases. For example, in the untapered case, as $z$ increases from $10 \mathrm{~m}$, the main-signal power in fact slightly decreases (because of degradation of electron bunching) while the sideband power increases. As $z>30 \mathrm{~m}$, the sideband power has become comparable to the main-signal power and more sideband spectral contents begin to emerge. It is expected that the linear theory is no longer valid. For the case of $10 \%$ undulator tapering, the saturation may be a bit delayed because the main signal can still grow, thanks to strong undulator tapering.

Having compared the sideband field gains, let us examine the evolution of FEL output spectra for the three different undulator taperings. From Fig. 5(a) and (b), both the main-signal power and sideband power with $0.8 \%$ taper ratio are comparable to those of the untapered case. Although being accompanied with increasing sideband power, the main-signal power with $10 \%$ taper ratio rises up to six times that of the untapered case. The increase of the main signal due to undulator tapering also results in the increase of synchrotron sideband frequency $\left[\Omega_{\text {syn }} \propto \sqrt{\left|\mathcal{E}_{0}\right|}\right.$, see Eq. (9)]. Thus the sampling synchrotron sideband frequencies (marked as thin red or blue lines for lower or upper sidebands, respectively) over the tapered FEL output spectrum are pushed outward compared with the untapered case. It can be even wider for larger taper ratio, as evidenced in Fig. 5(b) and (c), for both lower and upper sidebands. The thick red and blue lines in the subfigures are used to indicate the final synchrotron sideband frequency at the undulator exit. For the untapered case, the sampling synchrotron sideband frequency does in fact move back and forth within a certain sideband spectral range because of the periodic oscillation of the saturation power about an equilibrium (see also Fig. 3). The back-and-forth sampling will result in accumulation of sideband field gain. When 

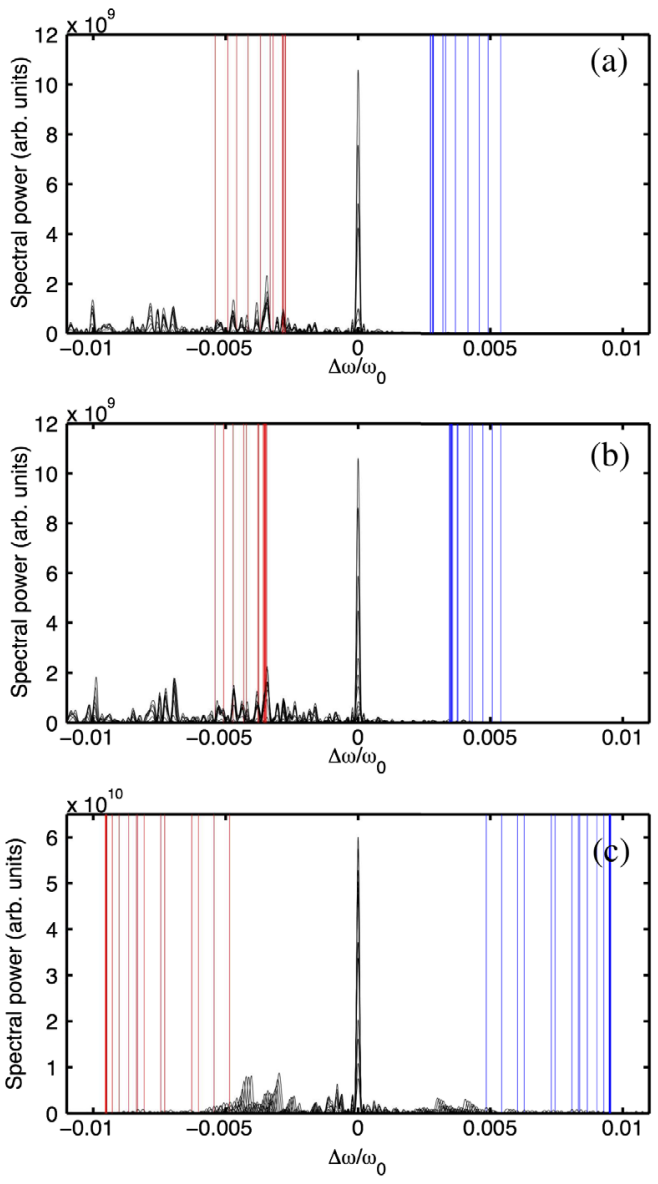

FIG. 5. FEL output spectra for (a) untapered, (b) 0.8\%-tapered, and (c) 10\%-tapered cases. Each of the spectra are overlapped at several equal-distance $z$ locations. The thin red and blue lines denote the lower and upper sampling synchrotron sideband frequencies, respectively. The thick red and blue lines indicate the final synchrotron sideband frequency sampled at the undulator exit.

increasing the ratio of undulator tapering, the sideband spectrum will broaden and the corresponding sideband field gain will be reduced. This numerical observation is consistent with the analysis in Ref. [13].

It has been known that due to hardware limitations, the amount of the taper ratio in LCLS undulator system can only be up to $0.8 \%$. We mentioned that the sideband growth can be reduced by increasing the taper ratio [Eq. (22)] (while the reduction will become ineffective as the taper ratio continues to increase). Using numerical simulations it can be found that 10\% [as shown in Fig. 4(c) and Fig. 5(c)] appears to be plausible for both maximizing the main signal and reducing the sideband signal powers.

Note that the present analysis of quantifying sideband field growth may have a limitation because the sampling synchrotron sideband frequencies are drifting, associated with varying or oscillating main-signal power. Besides, as previously mentioned, an electron beam consisting of different longitudinal slices will execute different phase

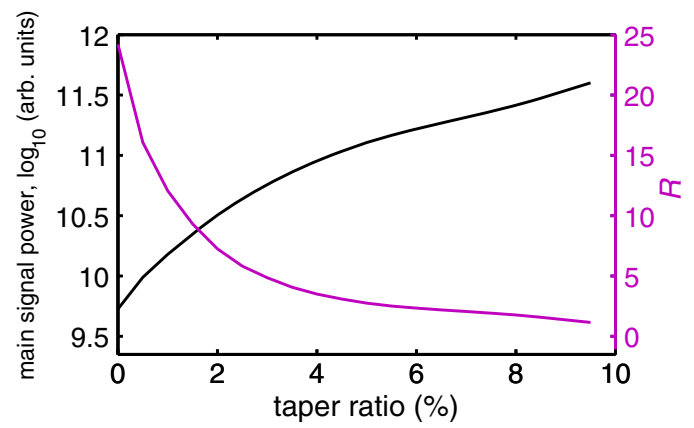

FIG. 6. The main signal (in logarithmic scale) and the ratio $R$ as a function of the taper ratio $\Delta$. The curves in this figure are obtained from the averaged results of 50 independent runs for each taper ratio.

space motions and thus respond to different portions of the sideband spectrum. An alternative way to quantify the contributions of sideband and main-signal spectral contents is to define the ratio $R$ as the spectral power outside the FEL gain bandwidth $2 \rho$ (of the main signal) to that within $2 \rho$. In general the larger this ratio is, the more contribution the sideband signal makes. Figure 6 shows the dependence of the main-signal power and the ratio $R$ as a function of the undulator tapering. Not shown here but a similar conclusion is also drawn when we use a three-dimensional numerical simulation [32].

As a side note, we have also applied the developed 1-D FEL sideband theory and simulations to the soft $\mathrm{x}$-ray regime, in particular for the soft x-ray self-seeding (SXRSS) case based on LCLS-like parameters. Assume a reference beam energy of $4.0 \mathrm{GeV}$, relative rms energy spread $1 \times 10^{-4}$, normalized transverse beam emittance $0.5 \mu \mathrm{m}$, peak current $1 \mathrm{kA}$, and other beam and undulator parameters the same as those listed in Table I. The FEL parameters are: the input seed power is assumed $20 \mathrm{~kW}$ and the resonant wavelength is $1.7422 \mathrm{~nm}$. The self-seeding chicane is located between the eighth and tenth undulator sections, i.e., the exit of the first undulator section (among a total of three big undulator sections). Let $z=0$ be at the entrance of tenth undulator section. The initial saturation occurs at $z \approx 15 \mathrm{~m}$. Assume still $0.8 \%$ undulator tapering and the same starting location of the previous hard $\mathrm{x}$-ray undulator configuration, which corresponds to $z \approx 36 \mathrm{~m}$ in the present coordinate setup. Our numerical simulation indicates that the sideband field gain reaches saturation $G(z) \approx e^{6} \approx 400$ at $z \approx 45 \mathrm{~m}$, which roughly corresponds to the twenty-fourth undulator segment. This numerical observation is consistent with what has been investigated in the experiment (see, for example, Ref. [33]).

\section{SUMMARY AND DISCUSSION}

In this paper we followed Refs. $[23,24]$ to study the FEL sideband effects based on similar parameters to those of the operating LCLS X-ray FEL and have investigated the 
dependence of the sideband instability growth rate on the undulator tapering. We derived the sideband dispersion equation based on 1-D high-gain tapered FEL model in the post-saturation regime, and further examined the approximate expressions for the maximum sideband growth rate. With emphasis on the gentle and strong tapering, we obtained Eqs. (22) and (23), respectively. In Eq. (22) we find that the undulator tapering can have a mitigating effect on sideband growth, through the direct taper ratio $\Delta$, while the increase of the main signal due to the undulator tapering will make the reduction become ineffective [Eq. (23) or Fig. 6]. Then we apply the theoretical predictions, including the semianalytical and numerical calculations, and compare with the full 1-D numerical FEL simulations based on the LCLS-like beam and undulator parameters. The sideband field is extracted from the FEL output radiation spectrum by accounting for the finite energy spread of the electron beam in the longitudinal phase space. Both the theoretical prediction and the 1-D numerical simulation show reasonably good agreement. The theory predicts that the sideband growth rate (measured at $z \approx 30 \mathrm{~m}$ about to sideband saturation) without undulator tapering and with $0.8 \%$ tapering will be comparable and about 3.8. The full 1-D numerical simulations indicate that both corresponding growth rates are about 4 , which is close to the theoretical predictions. For $10 \%$ undulator tapering, the theoretical prediction of the sideband growth rate matches well at the onset of sideband instability until $z \approx 15 \mathrm{~m}$, and gradually overestimates the result from numerical simulations at $z \approx 30 \mathrm{~m}$, as explained before. We have also provided an alternative quantification of sideband to main signal ratio by defining the spectral contents outside and inside the FEL gain bandwidth $2 \rho$. The dependence of this ratio $R$ on the undulator taper ratio $\Delta$ also indicates the relative reduction of sideband power due to strong undulator tapering.

The instantaneous growth rate obtained from Eq. (20) [or Eqs. (21)-(23)] can serve as a quick estimate for the FEL sideband effect. We have mentioned in Sec. I that the analysis begins at the first saturation where the information of the electron beam and radiation field should be provided. In case when a machine and the undulator system are in design status or the information at saturation is not yet available, to estimate the beam parameters and FEL performance at saturation we can apply the Ming-Xie fitting formula [34] for SASE mode or the simplified 1-D FEL amplifier theory (see, for example, Ref. [35]) for seeded FEL mode.

As an FEL sideband instability study in this paper, we note that the analysis is based upon single-particle description. That is to say, for a more general and realistic beam phase space distribution with finite energy spread, the approach adopted in this paper will be no longer valid and the theoretical prediction can become inaccurate. In that case we need to apply the Vlasov equation to properly treat the phase space dynamics. Furthermore the model we formulated is one-dimensional, which means that we have neglected the effect from the transverse dimension on the development of both the main signal and sideband fields. To be specific, we have assumed the electron beam size be much larger than the transverse extension of the radiation field in the 1-D theory. The diffraction effect and the lack of gain guiding [36] are not yet included in our analysis. In reality after the saturation the gain guiding becomes weaker and weaker, so that the confinement of the radiation field due to electron transverse density profile should be taken into account. Extending the present one-dimensional analysis to the two-dimensional case by solving the radial wave equation is planned.

\section{ACKNOWLEDGMENTS}

The authors would like to thank Dr. Chris Tennant of Jefferson Lab for reading this manuscript and for many helpful comments. They also thank Prof. Alex Chao and Dr. Yunhai Cai for stimulating discussions. The work was supported by the U.S. Department of Energy (DOE) under Contract No. DE-AC02-76SF00515 and the U.S. DOE Office of Science Early Career Research Program Grant No. FWP-2013-SLAC-100164.

[1] P. Emma et al., First lasing and operation of angstromwavelength free-electron laser, Nat. Photonics 4, 641 (2010).

[2] T. Ishikawa et al., A compact x-ray free-electron laser emitting in the sub-angstrom region, Nat. Photonics 6, 540 (2012).

[3] A. Aquila et al., The linac coherent light source single particle imaging road map, Struct. Dyn. 2, 041701 (2015).

[4] N. Kroll, P. Morton, and M. Rosenbluth, Free-electron lasers with variable parameter wigglers, IEEE J. Quantum Electron. 17, 1436 (1981).

[5] Y. Jiao, J. Wu, Y. Cai, A. W. Chao, W. M. Fawley, J. Frisch, Z. Huang, H.-D. Nuhn, C. Pellegrini, and S. Reiche, Modeling and multidimensional optimization of a tapered free electron laser, Phys. Rev. ST Accel. Beams 15, 050704 (2012).

[6] C. Emma, K. Fang, J. Wu, and C. Pellegrini, High efficiency, multiterawatt x-ray free electron lasers, Phys. Rev. Accel. Beams 19, 020705 (2016).

[7] J. Wu, N. Hu, H. Setiawan, X. Huang, T. O. Raubenheimer, Y. Jiao, G. Yu, A. Mandlekar, S. Spampinati, K. Fang, C. Chu, and J. Qiang, Multi-dimensional optimization of a terawatt seeded tapered free electron laser with a multiobjective genetic algorithm, Nucl. Instrum. Methods Phys. Res., Sect. A 846, 56 (2017).

[8] G. Geloni, V. Kocharyan, and E. Saldin, Scheme for generation of fully-coherent, TW power level hard X-ray pulses from baseline undulators at the European X-ray FEL, arXiv:1007.2743.

[9] E. A. Schneidmiller and M. V. Yurkov, Obtaining high degree of circular polarization at X-ray free electron lasers 
via a reverse undulator taper, Phys. Rev. ST Accel. Beams 16, 110702 (2013).

[10] R. C. Davidson and J. S. Wurtele, Single-particle analysis of the free-electron laser sideband instability for primary electromagnetic wave with constant phase and slowly varying phase, Phys. Fluids 30, 557 (1987).

[11] W. M. Sharp and S. S. Yu, Two-dimensional vlasov treatment of free-electron laser sidebands, Phys. Fluids B 2, 581 (1990).

[12] R. P. Pilla and A. Bhattacharjee, Elimination of the sideband instability in variableparameter freeelectron lasers and inverse freeelectron lasers, Phys. Plasmas 1, 390 (1994).

[13] S. Riyopoulos, Sideband suppression in tapered wiggler free electron lasers including thermal spreads, Phys. Plasmas 7, 1586 (2000).

[14] J. Masud, T. C. Marshall, S. P. Schlesinger, F. G. Yee, W. M. Fawley, E. T. Scharlemann, S. S. Yu, A. M. Sessler, and E. J. Sternbach, Sideband Control in a MillimeterWave Free-Electron Laser, Phys. Rev. Lett. 58, 763 (1987).

[15] F. G. Yee, J. Masud, T. C. Marshall, and S. P. Schlesinger, Power and sideband studies of a raman fel, Nucl. Instrum. Methods Phys. Res., Sect. A 259, 104 (1987).

[16] R. L. Tokar, B. D. McVey, and J. C. Goldstein, Sideband suppression in free electron lasers using a grating rhomb, IEEE J. Quantum Electron. 24, 856 (1988).

[17] D. C. Quimby, Sideband generation and mode structure in free-electron lasers, Proc. SPIE Int. Soc. Opt. Eng. 0738, 103 (1988).

[18] R. Lindberg, Report No. AOP-TN-2015-028, 2015.

[19] Z. Zhang, R. Lindberg, W. M. Fawley, Z. Huang, J. Krzywinski, A. Lutman, G. Marcus, and A. Marinelli, Microbunching-instability-induced sidebands in a seeded free-electron laser, Phys. Rev. Accel. Beams 19, 050701 (2016).

[20] E. Hemsing, B. Garcia, Z. Huang, T. Raubenheimer, and D. Xiang, Sensitivity of echo enabled harmonic generation to sinusoidal electron beam energy structure, Phys. Rev. Accel. Beams 20, 060702 (2017).

[21] P. R. Ribic, E. Roussel, G. Penn, G. De Ninno, L. Giannessi, G. Penco, and E. Allaria, Echo-enabled harmonic generation studies for the fermi free-electron laser, Photonics Spectra 4, 19 (2017).

[22] E. Roussel, E. Ferrari, E. Allaria, G. Penco, S. D. Mitri, M. Veronese, M. Danailov, D. Gauthier, and L. Giannessi, Multicolor High-Gain Free-Electron Laser Driven by
Seeded Microbunching Instability, Phys. Rev. Lett. 115, 214801 (2015).

[23] R. Bonifacio, F. Casagrande, M. Ferrario, P. Pierini, and N. Piovella, Hamiltonian model and scaling laws for freeelectron-laser amplifiers with tapered wiggler, Opt. Commun. 66, 133 (1988).

[24] S. Isermann and R. Graham, Suppression of the sideband instability in tapered free-electron lasers, Phys. Rev. A 45, 4050 (1992).

[25] R. L. Gluckstern, S. Krinsky, and H. Okamoto, Analysis of the saturation of a high-gain free-electron laser, Phys. Rev. E 47, 4412 (1993).

[26] S. Riyopoulos and C.M. Tang, The structure of the sideband spectrum in free electron lasers, Phys. Fluids 31, 1708 (1988).

[27] C. Penman and B.W. J. McNeil, Simulation of input electron noise in the free-electron laser, Opt. Commun. 90, 82 (1992).

[28] W. M. Fawley, Algorithm for loading shot noise microbunching in multidimensional, free-electron laser simulation codes, Phys. Rev. ST Accel. Beams 5, 070701 (2002).

[29] K.-J. Kim, Z. Huang, and R. Lindberg, Synchrotron Radiation and Free-Electron Lasers: Principles of Coherent X-ray Generation (Cambridge University Press, Cambridge, England, 2017).

[30] D. F. Ratner et al., FEL gain length and taper measurements at LCLS, Conference Proceedings TUOA03, 221 (2009).

[31] W. M. Fawley, Z. Huang, K.-J. Kim, and N. A. Vinokurov, Tapered undulators for sase fels, Nucl. Instrum. Methods Phys. Res., Sect. A 483, 537 (2002).

[32] S. Reiche, Genesis 1.3: a fully 3d time-dependent fel simulation code, Nucl. Instrum. Methods Phys. Res., Sect. A 429, 243 (1999).

[33] D. Ratner et al., Experimental Demonstration of a Soft X-Ray Self-Seeded Free-Electron Laser, Phys. Rev. Lett. 114, 054801 (2015).

[34] M. Xie, Design optimization for an X-ray free electron laser driven by SLAC LINAC, Conference Proceedings C950501, 183 (1996).

[35] E. L. Saldin, E. V. Schneidmiller, and M. V. Yurkov, The Physics of Free Electron Lasers (Springer-Verlag, Berlin Heidelberg, 2000).

[36] Z. Huang and K.-J. Kim, Transverse and temporal characteristics of a high-gain free-electron laser in the saturation regime, Nucl. Instrum. Methods Phys. Res., Sect. A 483, 504 (2002). 